\title{
Repetition Time
}

National Cancer Institute

\section{Source}

National Cancer Institute. Repetition Time. NCI Thesaurus. Code C82330.

The amount of time in milliseconds between successive pulse sequences applied to the same slice. 